\title{
Inter-Individual Responses to Citrulline Malate Oral Supplementation on Post-Exercise Hypotension in Hypertensives: A 24-Hour Analysis
}

\author{
Juliano Casonatto, ${ }^{\circledR}$ Daniel Massaharu Enokida, ${ }^{\circledR}$ Kamila Grandolfi \\ Universidade Norte do Paraná, Londrina, PR - Brazil
}

\begin{abstract}
Background: Studies have persuasively demonstrated that citrulline has a key role in the arginine-nitric oxide system, increasing nitric oxide bioavailability, an important mediator of peripheral vasodilation.
\end{abstract}

Objective: To analyze the inter-individual post-exercise hypotension responsiveness following acute citrulline supplementation in hypertensives.

Methods: Forty hypertensives were randomly assigned to one of the four experimental groups (control-placebo, control-citrulline, exercise-placebo, and exercise-citrulline). They ingested placebo or citrulline malate [CM] (6 grams). During the exercise session, individuals performed 40 minutes of walking/running on a treadmill at $60-70 \%$ of $\mathrm{HR}$ reserve. For the control session, the individuals remained seated at rest for $\mathbf{4 0}$ minutes. Office blood pressure (BP) was taken every 10 minutes until completing 60 minutes after the experimental session. The ambulatory BP device was programmed to take the readings every 20 minutes (awake time) and every 30 minutes (sleep time) over the course of 24 hours of monitoring. Statistical significance was defined as $p<0.05$.

Results: Unlike the other experimental groups, there were no "non-responders" in the exercise/citrulline (EC) for "awake" (systolic and diastolic BP) and "24 hours" (diastolic BP). The effect sizes were more consistent in the EC for systolic and diastolic ambulatorial BP response. The effects were "large" (> 0.8) for "awake", "asleep", and "24 hours" only in the EC for diastolic BP.

Conclusion: CM supplementation can increase the post-exercise hypotensive effects in hypertensives. In addition, the prevalence of non-responders is lower when associated with aerobic exercise and CM supplementation. (Arq Bras Cardiol. 2019; 113(2):218-228)

Keywords: Exercise; Hypertension; Blood Pressure Monitoring Ambulatory; Citruline; Amino Acids; Nitric Oxide; Dietary Supplements.

\section{Introduction}

Post-exercise hypotension (PEH) is defined as a sustained reduction in blood pressure (BP) after a single bout of exercise. ${ }^{1}$ This transient reduction can last up to $22 \mathrm{~h}$ after completion of an exercise session. ${ }^{2}$ For this reason, $\mathrm{PEH}$ is now considered to be an important physiological phenomenon, ${ }^{1}$ which can play a major role in BP management. It is important to emphasize that $\mathrm{PEH}$ is a physiological phenomenon characterized by a reduction in BP following exercise compared with pre-exercise or control session values, with may be sustained for some hours. ${ }^{3}$ Therefore, for the PEH to be clinically significant, an important magnitude and maintenance of this reduction for some hours are necessary. Furthermore, previous studies

Mailing Address: Juliano Casonatto •

Universidade Norte do Paraná - Rua Marselha, 591. Postal Code 86041-140, Londrina, PR - Brazil

E-mail: juliano2608@hotmail.com

Manuscript received August 28, 2018, revised manuscript October 29, 2018, accepted November 14, 2018

DOI: $10.5935 / a b c .20190115$ have reported that the magnitude of $\mathrm{PEH}$ following a single bout of exercise was correlated with chronic changes in rest BP after a period of exercise training. ${ }^{4-8}$

However, despite this possible relationship, some individuals present $\mathrm{PEH}$ (i.e., "responders") while others do not (i.e., "non-responders"). Past studies have identified "responders" and "non-responders" in non-hypertensive. , $^{, 8-10}$ and hypertensive populations. ${ }^{5,6,11}$ This means that, in the same intervention group, some individuals may demonstrate $\mathrm{PEH}$ whereas others show no changes or even an increased BP response to exercise training. Indeed, results from $\mathrm{PEH}$ studies are generally limited to presenting the mean BP reduction responses of the training group, ignoring inter-individual variations which can lead to misinterpretation, as positive effects from a given exercise training protocol may not be fully applied for each person individually. ${ }^{12}$

On the other hand, studies have persuasively demonstrated that citrulline (a non-essential amino acid) has a key role in the arginine-nitric oxide system, increasing nitric oxide (NO) bioavailability, ${ }^{13}$ an important mediator of peripheral vasodilation. Thus, it is possible that $\mathrm{PEH}$ could be more pronounced after citrulline supplementation, especially in hypertensives, since the BP responses would be different as $\mathrm{PEH}$ can be caused by different mechanisms between normotensive 
and hypertensive individuals. Previous studies have shown that hypertensive individuals present cardiac output reduction, ${ }^{2,14}$ while normotensive individuals present a reduction in total peripheral resistance. ${ }^{14-16}$ For this reason, a non-pharmacological vasodilatation strategy can help hypertensive patients to activate another PEH mechanism together with a cardiac output decrease resulting in additional $\mathrm{PEH}$ effects.

Other experiments have observed that the plasma and salivary $\mathrm{NO}$ were associated with $\mathrm{PEH}$ after resistance ${ }^{17}$ and aerobic exercises ${ }^{17-20}$ in subjects with chronic diseases. Additionally, citrulline supplementation has shown promise with pre-clinical (animal) evidence for atherogenicendothelial protection and preliminary evidence is also available for citrulline-induced benefits to muscle and metabolic health (via vascular and non-vascular pathways) in susceptible/older populations. ${ }^{21}$

For these reasons, this study aimed to analyze the inter-individual $\mathrm{PEH}$ responsiveness following an acute citrulline supplementation in hypertensives. Our hypotheses were: i) there would be an inter-individual variability in BP responsiveness following a single bout of aerobic exercise and ii) citrulline oral supplementation could contribute to reducing the rate of non-responders.

\section{Methods}

\section{Participants}

After sample size calculation (see statistical analysis session), 40 hypertensive, sedentary individuals participated in the study. All volunteers (both sexes) were adults without osteoarticular disabilities and had medical authorization to practice physical exercise. Participants were recruited from an exercise program project linked to the university that offered stretching and functional exercise sessions to the external community. The study followed the Declaration of Helsinki and the Institutional Ethics
Committee approved all experimental procedures and protocols (78697617.4.0000.0108). Each participant was fully informed of all potential risks and experimental procedures, after which, informed written consent was obtained. Table 1 shows the basic characteristics of the participants.

\section{Study design}

A double-blind, placebo-controlled, parallel-groups clinical trial was performed (Figure 1). The participants were randomly allocated (using a random number table - https://www. random.org/) into four different experimental groups (exercise/ citrulline [EC]; exercise/placebo [EP]; control/citrulline [CC]; control/placebo $[\mathrm{CP}])$. The participants ingested a sachet, which contained citrulline malate (CM) (6 grams) or placebo (6 grams of corn starch) dissolved in water.

The substances were ingested 120 minutes before the experimental or control session. Anthropometric measures were taken before the rest period. The exercise session consisted of a 5 min warm up ( $50 \%$ of $65 \%$ HR [heart rate] reserve) and 40 minutes of running/walking at $60-70 \% \mathrm{HR}$ reserve on a treadmill. This was followed by a progressive cooldown (5 minutes). The exercise intensity was also evaluated based on the Borg ${ }^{22}$ Rating of Perceived Exertion (RPE 6-20 score). The RPE was collected every 5 minutes over the course of 40 minutes during the running/walking exercise period. The exercise intensity was reduced if the participant reported $\mathrm{RPE} \geq 15$, independently of HR. In the control session, the participants remained seated in a quiet room for $40 \mathrm{~min}$.

After the exercise/control sessions, the BP was measured every 10 minutes over the course of 60 minutes (laboratory phase). Next, the participants were allowed 15 minutes to take a shower and change their clothes before the ambulatory BP device was attached on their arm. The ambulatory BP was recorded over 24 hours. The participants were asked to return to the laboratory the next day to remove the device.

Table 1 - Characteristics of participants and antihypertensive medications

\begin{tabular}{|c|c|c|c|c|c|c|c|c|c|c|}
\hline & \multicolumn{2}{|c|}{ Control-Placebo } & \multicolumn{2}{|c|}{ Control-Citrulline } & \multicolumn{2}{|c|}{ Exercise-Placebo } & \multicolumn{2}{|c|}{ Exercise-Citrulline } & \multirow{2}{*}{$F$} & \multirow{2}{*}{$p$} \\
\hline & Mean & SD & Mean & SD & Mean & SD & Mean & SD & & \\
\hline Age (years) & 62.3 & 18.7 & 60.6 & 16.8 & 52 & 15.2 & 58.6 & 8.6 & 0.864 & 0.469 \\
\hline Weight (kg) & 77.2 & 16.5 & 76.5 & 9.0 & 79.6 & 17.2 & 72.5 & 13.1 & 0.419 & 0.740 \\
\hline Height (m) & 1.66 & 0.11 & 1.59 & 0,08 & 1.61 & 0.08 & 1.58 & 0.09 & 1.309 & 0.286 \\
\hline BMI & 27.9 & 4.2 & 30.6 & 4.6 & 30.8 & 6.5 & 29.1 & 5.8 & 0.625 & 0.603 \\
\hline \multirow[t]{2}{*}{ WC (cm) } & 98.2 & 9.9 & 101.4 & 9.1 & 98.5 & 14.9 & 99.1 & 11.1 & 0.159 & 0.923 \\
\hline & $\mathbf{N}$ & $\%$ & $\mathbf{N}$ & $\%$ & $\mathbf{N}$ & $\%$ & $\mathbf{N}$ & $\%$ & & \\
\hline BB & 3 & 30 & 5 & 50 & 5 & 50 & 3 & 30 & & \\
\hline ACEI & 5 & 50 & 3 & 30 & 4 & 40 & 6 & 60 & & \\
\hline DIUR & 0 & 0 & 1 & 10 & 0 & 0 & 1 & 10 & & \\
\hline ACEI+DIUR & 0 & 0 & 0 & 0 & 0 & 0 & 0 & 0 & & \\
\hline $\mathrm{BB}+\mathrm{ACE}+\mathrm{DIUR}$ & 0 & 0 & 0 & 0 & 1 & 10 & 0 & 0 & & \\
\hline
\end{tabular}

SD: standard deviation; BMI: body mass index; WC: waist circumference; BB: beta-blockers; ACEl: angiotensin-converting-enzyme inhibitor; DIUR: diuretics. 
The study protocol was registered in ClinicalTrials.gov (NCT03378596).

\section{Anthropometry}

The weight was measured by a digital anthropometric scale (Urano, OS 180A, Canoas, Brazil), with an accuracy of $0.1 \mathrm{~kg}$ and height was measured by a stadiometer with an accuracy of $0.1 \mathrm{~cm}$, in accordance with the procedures described by Gordon et al. ${ }^{23}$ The body mass index was defined as the body mass $(\mathrm{kg})$ divided by the square of the body height.

\section{Office blood pressure (laboratorial phase)}

The office BP measurements were taken with an oscillometric device (Omron MX3 Plus, Bannockburn, USA) previously validated for clinical measures in adults. ${ }^{24}$ Firstly, the participants remained seated (rest period) in a calm, quiet, and thermoneutral $\left(22^{\circ}-24^{\circ} \mathrm{C}\right)$ environment for $20 \mathrm{~min}$. BP was measured three times during the rest period (at $10 \mathrm{~min}, 15 \mathrm{~min}$, and $20 \mathrm{~min}$ ). The rest BP value was considered as the average of these three measurements. Immediately following the sessions (exercise or control), BP was measured in a quiet environment for 60 minutes. The BP measurements were taken according to the American Heart Association recommendations. ${ }^{25}$

Ambulatorial blood pressure measures (ambulatorial phase)

The ambulatorial BP measurements were taken with an oscillometric device (Dyna-MAPA - São Paulo, Brazil) attached on the left arm, always by the same investigator, in accordance with procedures described by the American Heart Association. ${ }^{25}$ The participants received instructions to keep their arm extended during the measures. The device was calibrated by direct comparison with a mercury sphygmomanometer, by a trained technical person, in agreement with recommendations. ${ }^{25}$

The monitor was set to register the systolic and diastolic BP and HR every 20 minutes during "daytime" (08:00 am to $11: 00 \mathrm{pm})$ and every 30 minutes during "night-time" (11:00 pm to 08:00 am) to reduce sleep disturbances. The device screen was electronically blinded to avoid feedback. All participants were instructed to register and report their sleep time in a diary on the following day.

The data were recorded in the device memory and then sent to a computer using specific software (Aplicação Dyna Mapa - Version 5.0.382.12) for analysis. The average of the valid readings was above $90 \%$ for all participants.

\section{Statistical analysis}

Assuming a standard deviation (SD) of $5 \mathrm{mmHg}^{26}$ for the systolic BP, an alpha of $5 \%$ and a desired statistical power of $80 \%$ for detecting a minimum difference of $7 \mathrm{mmHg}{ }^{26}$ 7 subjects were required in each group. The Shapiro-Wilk test was employed to examine the normality of the data distribution. The data are presented in the text as mean and SD. Levene's homogeneity test was applied before the ANOVA one-way. Turkey (if homogeneity was accepted) or Games-Howell (if homogeneity was not accepted) multiple comparisons were employed to examine differences between pairs of trials. Effect size from the paired t-test was calculated
( $d=$ mean/SD). The results of the periods (60 minutes, awake, asleep, and 24 hours) were obtained through the average of the respective periods. Statistical significance was defined as $p<0.05$.

To assess the inter-individual BP responsiveness, we first assessed the reproducibility of pre-exercise BP between " -10 " and " 0 " minutes of rest BP measures (see Figure 1). For this analysis, the intraclass correlation coefficient (ICC, model $2, \mathrm{k}$ ), coefficient of variation (CV) and standard error measurements (SEM) were considered. The CV between pre-exercise BP measurements was calculated as follows: $C V=100 \times\left(2 \times\left(S_{d} / \sqrt{ }^{2}\right) /\left(X_{1}+X_{2}\right) . S D_{d}\right.$ represents the $S D$ of the differences between the 2 measurements, and $X_{1}$ and $X_{2}$ represent the 2 measurement means, respectively. $\mathrm{SEM}=\mathrm{SDx} \sqrt{(1-I C C)}$, with $\mathrm{SD}$ representing the $\mathrm{SD}$ of the first pre-exercise BP measure. The SEM was used as a measure of variability, but primarily for the minimal detectable change (MDC) calculation. MDC, which is the minimal change necessary to provide confidence that the results are not a random variation or measurement error, was calculated as follows: $\mathrm{MDC}=\mathrm{z}$-score $(95 \% \mathrm{Cl})$ $x S E M x \sqrt{ } 2$. All above-mentioned procedures were adopted according to Haley and Fragala-Pinkham ${ }^{27}$ and Darter et al. ${ }^{28}$ The participants were considered as "responder" if their BP reached a value equal to or greater than the MDC.

The statistical analysis was generated using the SPSS, version 20 , system for windows.

\section{Results}

The exercise intensity was reduced for three participants (15 [EC], 18 and 19 [EP]) because they reported RPE $\geq 15$. The exercise intensity based on HR reserve varied between $51-59 \%$ during the time ( $9 \pm 3$ minutes) that they could not keep $60-70 \%$ HR reserve.

The results of the variables related to the MDC calculation were: 0.915 (ICC), 5.08 (CV), 5.25 (SEM) and 2.37 (MDC) for systolic BP, and 0.846 (ICC), 5.64 (CV), 3.74 (SEM) and 1.69 (MDC) for diastolic BP.

Table 2 presents the absolute and relative BP changes in the different experimental groups. A significant reduction in systolic BP was identified for the EC when compared with the $\mathrm{CP}$ in the first 60 minutes (relative changes) after the experimental session. The same lowering effects were observed over the course of 24 hours (absolute changes) and for the "awake" and "24 hour" periods, considering relative changes ( $\triangle \%$ Awake and $\Delta \% 24$ hours) compared to the CP. No significant differences were found for the CC and EP.

Table 3 presents the effect sizes from the paired t-test (rest vs $60 \mathrm{~min}$, awake, asleep, and 24 hours) for each experimental group. Considering systolic BP, the $\mathrm{CP}$ showed a significant effect for the asleep and 24 hours, the EP showed a significant effect for the awake and asleep, and the EC showed a significant effect for all periods (60 min, awake, asleep, and 24 hours). Additionally, considering diastolic BP, the $\mathrm{CP}$ presented a significant effect for the asleep, the CC presented a significant effect for the $60 \mathrm{~min}$, asleep, and 24 hours, the EP presented a significant effect for the awake and asleep, and the EC presented a significant effect for the awake, asleep, and 24 hours. 


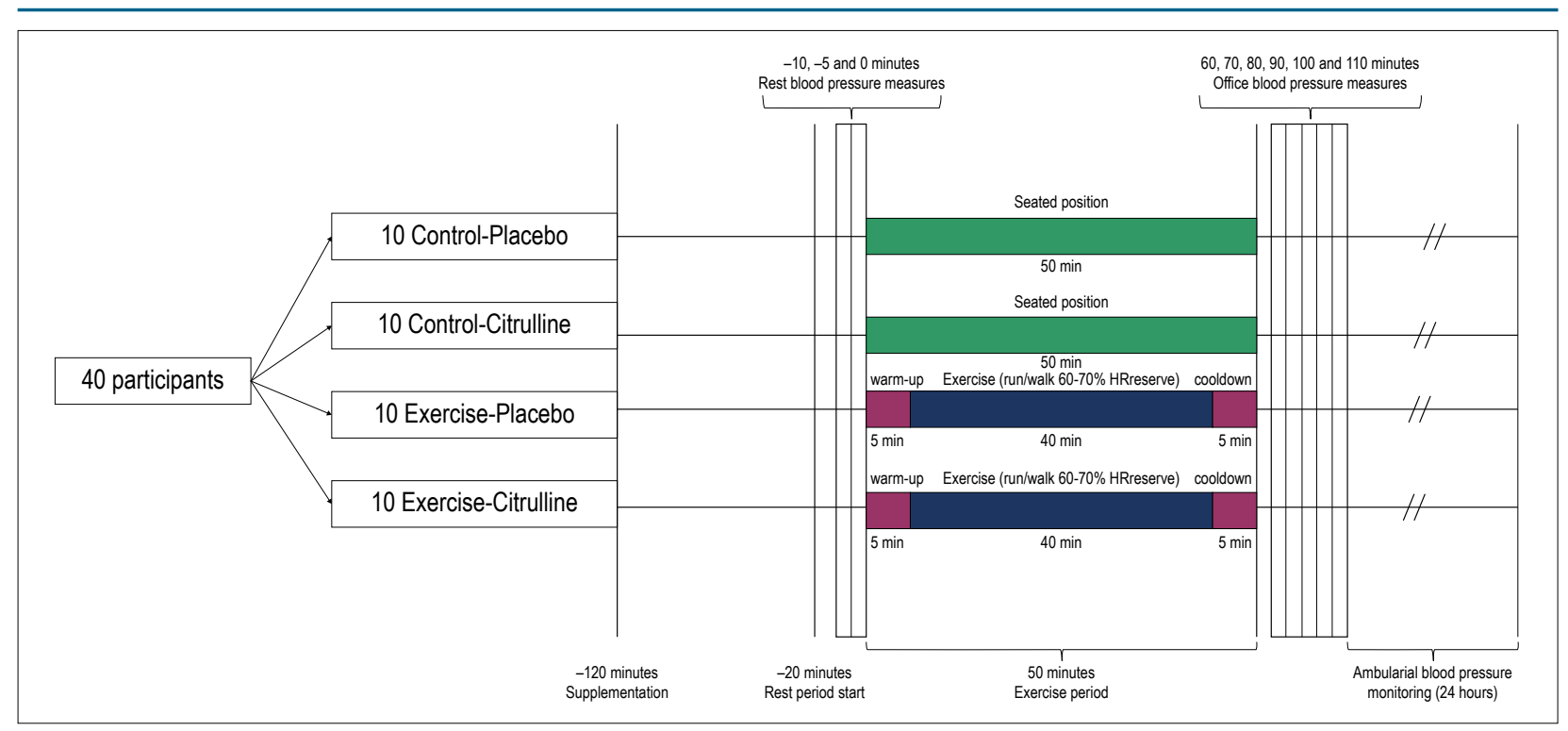

Figure 1 - Study design.

Table 2 - Absolute (rest, $60 \mathrm{~min}$, awake, asleep, and 24 hours) and relative ( $\Delta \% 60 \mathrm{~min}, \Delta \%$ Awake, $\Delta \%$ Asleep, $\Delta \% 24 \mathrm{hours}$ ) blood pressure changes

\begin{tabular}{|c|c|c|c|c|c|c|c|c|c|c|}
\hline & \multicolumn{2}{|c|}{ Control-Placebo } & \multicolumn{2}{|c|}{ Control-Citrulline } & \multicolumn{2}{|c|}{ Exercise-Placebo } & \multicolumn{2}{|c|}{ Exercise-Citrulline } & \multirow{2}{*}{$F$} & \multirow{2}{*}{$p$} \\
\hline & Mean & SD & Mean & SD & Mean & SD & Mean & SD & & \\
\hline \multicolumn{11}{|l|}{ SBP } \\
\hline Rest (mmHg) & 140 & 19 & 132 & 15 & 136 & 12 & 142 & 20 & 0.714 & 0.550 \\
\hline $60 \mathrm{~min}(\mathrm{mmHg})$ & 138 & 19 & 137 & 14 & 130 & 9 & 127 & 15 & 1.240 & 0.310 \\
\hline Awake (mmHg) & 127 & 11 & 128 & 8 & 124 & 11 & 126 & 8 & 0.334 & 0.801 \\
\hline Asleep (mmHg) & 125 & 16 & 120 & 14 & 126 & 10 & 126 & 15 & 0.465 & 0.709 \\
\hline 24 hours $(\mathrm{mmHg})$ & 128 & 11 & 125 & 9 & 127 & 10 & 125 & 13 & 0.216 & 0.884 \\
\hline$\Delta \% 60 \min$ & -2.3 & 3.4 & 5.4 & 9.4 & -6.3 & 11.4 & $-15.0^{* *}$ & 8.1 & 9.737 & $<0.001$ \\
\hline$\Delta \%$ Awake & -9.7 & 12.5 & -4.2 & 12.3 & -9.1 & 15.2 & -21.0 & 16.7 & 2.469 & 0.078 \\
\hline$\Delta \%$ Asleep & -15.8 & 14.0 & -9.8 & 16.2 & -9.5 & 19.5 & -17.0 & 14.1 & 0.588 & 0.627 \\
\hline$\Delta \% 24$ hours & -11.5 & 12.4 & -6.0 & 12.6 & -9.2 & 16.3 & -20.1 & 16.8 & 1.935 & 0.141 \\
\hline \multicolumn{11}{|l|}{ DBP } \\
\hline Rest (mmHg) & 82 & 5 & 80 & 9 & 86 & 11 & 85 & 10 & 0.889 & 0.456 \\
\hline $60 \mathrm{~min}(\mathrm{mmHg})$ & 83 & 9 & 84 & 8 & 86 & 10 & 85 & 10 & 0.237 & 0.870 \\
\hline Awake $(\mathrm{mmHg})$ & 80 & 8 & 75 & 8 & 79 & 7 & 71 & 9 & 2.847 & 0.051 \\
\hline Asleep (mmHg) & 75 & 11 & 70 & 8 & 76 & 10 & 71 & 10 & 1.083 & 0.369 \\
\hline 24 hours (mmHg) & 82 & 8 & 73 & 7 & 79 & 8 & $71^{*}$ & 9 & 3.999 & 0.015 \\
\hline$\Delta \% 60 \mathrm{~min}$ & 0.4 & 5.9 & 4.1 & 4.8 & 1.0 & 7.2 & 0.1 & 7.4 & 0.796 & 0.504 \\
\hline$\Delta \%$ Awake & -1.5 & 5.2 & -4.7 & 7.3 & -6.8 & 8.1 & $-13.9 \dagger$ & 6.2 & 5.917 & 0.002 \\
\hline$\Delta \%$ Asleep & -6.6 & 9.1 & -10.0 & 8.0 & -9.2 & 11.1 & -13.2 & 7.3 & 0.923 & 0.439 \\
\hline$\Delta \% 24$ hours & -3.3 & 6.1 & -6.8 & 6.9 & -7.6 & 8.4 & $-14.5^{*}$ & 6.4 & 4.505 & 0.009 \\
\hline
\end{tabular}

SD: standard deviation; SBP: systolic blood pressure; DBP: diastolic blood pressure; $\triangle \%$ : +- percent change from "rest" value. *: $p<0.05$ vs control-placebo (Tukey post-hoc); ${ }^{* *}: p<0.05$ vs control-placebo and control-citrulline (Games-Howell post-hoc); $\uparrow: p<0.05$ vs. control-placebo and control-citrulline (Tukey post-hoc). 
Table 3 - Effect size from Paired $t$ test (versus rest $[\mathrm{d}=$ mean/SD $]$ )

\begin{tabular}{|c|c|c|c|c|c|c|c|c|}
\hline & \multicolumn{2}{|c|}{ Control-Placebo } & \multicolumn{2}{|c|}{ Control-Citrulline } & \multicolumn{2}{|c|}{ Exercise-Placebo } & \multicolumn{2}{|c|}{ Exercise-Citrulline } \\
\hline & ES & $p$ & ES & $p$ & ES & $p$ & ES & $p$ \\
\hline \multicolumn{9}{|l|}{ SBP } \\
\hline $60 \mathrm{~min}$ & 0.61 & 0.084 & -0.56 & 0.109 & 0.53 & 0.127 & 1.81 & $<0.001$ \\
\hline Awake & 0.69 & 0.055 & 0.26 & 0.428 & 1.00 & 0.011 & 0.74 & 0.042 \\
\hline Asleep & 0.73 & 0.044 & 0.65 & 0.068 & 0.83 & 0.027 & 0.78 & 0.034 \\
\hline 24 hours & 0.92 & 0.017 & 0.48 & 0.156 & 0.57 & 0.102 & 0.88 & 0.020 \\
\hline \multicolumn{9}{|l|}{ DBP } \\
\hline $60 \mathrm{~min}$ & -0.12 & 0.717 & -0.91 & 0.018 & -0.12 & 0.707 & -0.01 & 0.968 \\
\hline Awake & 0.32 & 0.333 & 0.71 & 0.051 & 0.81 & 0.030 & 2.06 & $<0.001$ \\
\hline Asleep & 0.84 & 0.027 & 1.21 & 0.004 & 0.90 & 0.019 & 2.16 & $<0.001$ \\
\hline 24 hours & 0.04 & 0.905 & 0.97 & 0.014 & 0.65 & 0.071 & 2.27 & $<0.001$ \\
\hline
\end{tabular}

SD: standard deviation; ES: effect-size; SBP: systolic blood pressure; DBP: diastolic blood pressure.

The percentages of responders and non-responders in the different experimental groups for different time periods (60 min, awake, asleep, and 24 hours) are shown in table 4. The percentages of the systolic BP responders varied from $20 \%$ (CC) to $90 \%$ (EC) for $60 \mathrm{~min} ; 60 \%$ (CC and EP) to $100 \%$ (EC) for awake; $60 \%$ (CC and EP) to $90 \%$ (EC) for asleep; and $60 \%$ (CC and EP) to $90 \%$ (EC) for 24 hours.

The percentages of the diastolic $\mathrm{BP}$ responders varied from $10 \%$ (CC) to $40 \%$ (CP) for $60 \mathrm{~min}$; $50 \%$ (CP) to $100 \%$ (EC) for awake; $70 \%$ (CP) to $100 \%$ (CC and EC) for asleep; and $60 \%$ (CP) to $100 \%$ (EC) for 24 hours.

Absolute systolic and diastolic BP individual changes for each experimental group in the $60 \mathrm{~min}$, awake, asleep, and 24 hours are presented in Figures 1 (systolic) and 2 (diastolic). All participants in the $\mathrm{EC}$ demonstrated a reduction in systolic $\mathrm{BP}$ in the $60 \mathrm{~min}$, awake, and 24 hours. For the asleep period, only one EC participant (number 23) did not present a reduction in systolic BP (Figure 2).

All participants in the $\mathrm{EC}$ presented a reduction in diastolic BP in the awake, asleep, and 24 hours (Figure 3).

\section{Discussion}

The main findings of this study were: (i) there was considerable inter-individual responsiveness variability in systolic and diastolic BP responses following all experimental protocols, (ii) despite the inter-individual responsiveness variability, there was $\mathrm{PEH}$ only when associated with $\mathrm{CM}$ supplementation. To the best of our knowledge, this is the first report on inter-individual analysis of PEH following a single bout of aerobic exercise preceded by $\mathrm{CM}$ supplementation in hypertensive subjects.

Unlike the other experimental groups, there were no "non-responders" in the EC for "awake" (systolic and diastolic BP) and "24 hours" (diastolic BP). These results are interesting since "non-responders" have generally been identified both in non-hypertensive ${ }^{9,10}$ and hypertensive $e^{5,6}$ populations. It is important to clarify that in an intervention group, some individuals may demonstrate improvement in a given outcome (responder) whereas others show no changes or even an adverse response to exercise training (non-responders or adverse responders). ${ }^{9}$ In fact, the heterogeneity of responses to exercise training is a current concern in exercise investigation $s^{29}$ since even homogeneous samples may demonstrate a wide range of heterogeneous responses. ${ }^{12}$ In addition, results of $\mathrm{PEH}$ are generally limited to presenting the average reductions in the experimental group, ignoring inter-individual variations ${ }^{12}$ which can lead to misinterpretation since not all individuals respond favorably.

It is important to consider that the results reported by central tendency measures can vary depending on the analysis. In medical studies, it is common to take measurements before and after medical interventions. How to measure the changes from baseline is a common question posed by researchers. For example, in the present study, when absolute values were used for inter-group comparisons (Table 2), no statistical differences were found for systolic BP. On the other hand, using the percentage difference (delta), a significant

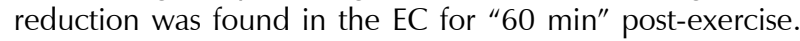
Another inconsistency can be seen in diastolic BP analysis. When absolute values were used for inter-group comparisons, a significant difference was found in the EC for "24 hours" post-exercise. On the other hand, using the percentage difference, significant reductions were found in the EC for "awake" and "24 hours" post-exercise. In specific situations, some studies (e.g., Vickers) ${ }^{30}$ suggested avoiding using percentage changes. Despite these inconsistencies, clinicians may prefer to choose the method that will most obviously demonstrate the health-improvement. Some researchers may choose the method that can be best understood by the majority of people interested in the research.

However, one of the most common questions driving the evaluation of intervention programs is "how does this effect compare with the effects of other interventions?". Therefore, previous studies have encouraged the use of "effect size" in quantitative studies. ${ }^{31}$ Researchers are often stimulated 
Table 4 - Percentage of responders and non-responders and inter-individual percentage changes ( $\mathrm{min}$; $\mathrm{max}$ ) on Control-Placebo, Control-Citrulline, Exercise-Placebo and Exercise-Citrulline groups

\begin{tabular}{|c|c|c|c|c|c|c|c|c|}
\hline & \multicolumn{2}{|c|}{ Control-Placebo } & \multicolumn{2}{|c|}{ Control-Citrulline } & \multicolumn{2}{|c|}{ Exercise-Placebo } & \multicolumn{2}{|c|}{ Exercise-Citrulline } \\
\hline & $\mathrm{N}$ & $\operatorname{Min}: \operatorname{Max}(\%)$ & $\mathrm{N}$ & $\operatorname{Min}: \operatorname{Max}(\%)$ & $\mathbf{N}$ & Min:Max (\%) & $\mathrm{N}$ & Min:Max (\%) \\
\hline \multicolumn{9}{|l|}{ SBP } \\
\hline \multicolumn{9}{|l|}{$60 \mathrm{~min}$} \\
\hline Responders & 5 & $(-2 ;-7)$ & 2 & $(-2 ;-4)$ & 7 & $(-2 ;-17)$ & 9 & $(-2 ;-18)$ \\
\hline Non-responders & 5 & $(-1 ; 2)$ & 8 & $(-1 ; 17)$ & 3 & $(0 ; 10)$ & 1 & $(-1 ;-1)$ \\
\hline \multicolumn{9}{|l|}{ Awake } \\
\hline Responders & 8 & $(-2 ;-18)$ & 6 & $(-4 ;-16)$ & 6 & $(-9 ;-24)$ & 10 & $(-3 ;-33)$ \\
\hline Non-responders & 2 & $(0 ; 9)$ & 4 & $(5 ; 16)$ & 4 & $(2 ; 13)$ & 0 & - \\
\hline \multicolumn{9}{|l|}{ Asleep } \\
\hline Responders & 8 & $(-5 ;-21)$ & 6 & $(-2 ;-33)$ & 6 & $(-8 ;-32)$ & 9 & $(-3 ;-28)$ \\
\hline Non-responders & 2 & $(4 ; 7)$ & 4 & $(-1 ; 9)$ & 4 & $(0 ; 13)$ & 1 & $(1 ; 1)$ \\
\hline \multicolumn{9}{|l|}{24 hours } \\
\hline Responders & 8 & $(-5 ;-18)$ & 6 & $(-2 ;-20)$ & 6 & $(-6 ;-26)$ & 9 & $(-2 ;-31)$ \\
\hline Non-responders & 2 & $(0 ; 9)$ & 4 & $(-1 ; 14)$ & 4 & $(1 ; 11)$ & 1 & $(-1 ;-1)$ \\
\hline \multicolumn{9}{|l|}{ DBP } \\
\hline \multicolumn{9}{|l|}{$60 \mathrm{~min}$} \\
\hline Responders & 4 & $(-3 ;-13)$ & 1 & $(-7 ;-7)$ & 3 & $(-3 ;-10)$ & 3 & $(-3 ;-13)$ \\
\hline Non-responders & 6 & $(0 ; 12)$ & 9 & $(1 ; 14)$ & 7 & $(-1 ; 18)$ & 7 & $(-2 ; 22)$ \\
\hline \multicolumn{9}{|l|}{ Awake } \\
\hline Responders & 5 & $(-3 ;-13)$ & 7 & $(-2 ;-19)$ & 6 & $(-3 ;-21)$ & 10 & $(-8 ;-27)$ \\
\hline Non-responders & 5 & $(-1 ; 10)$ & 3 & $(0 ; 10)$ & 4 & $(-1 ; 1)$ & 0 & - \\
\hline \multicolumn{9}{|l|}{ Asleep } \\
\hline Responders & 7 & $(-6 ;-30)$ & 10 & $(-1 ;-31)$ & 8 & $(-3 ;-28)$ & 10 & $(-6 ;-28)$ \\
\hline Non-responders & 3 & $(2 ; 5)$ & 0 & - & 2 & $(6 ; 6)$ & 0 & - \\
\hline \multicolumn{9}{|l|}{24 hours } \\
\hline Responders & 6 & $(-3 ;-15)$ & 8 & $(-1 ;-20)$ & 8 & $(-3 ;-22)$ & 10 & $(-7 ;-25)$ \\
\hline Non-responders & 4 & $(-2 ; 6)$ & 2 & $(5 ; 5)$ & 2 & $(-2 ; 2)$ & 0 & - \\
\hline
\end{tabular}

SBP: systolic blood pressure; DBP: diastolic blood pressure.

to report effect sizes for three reasons. First, to present the magnitude of the reported effects in a standardized metric; second, effect sizes allow researchers to draw meta-analytic conclusions by comparing standardized effect sizes across studies, and third, effect sizes from previous studies can be used when planning a new study, providing an indication of the average sample size needed. ${ }^{32}$ In the present study, we found that the effect sizes (from the paired t-test [versus rest]) were more consistent in the $\mathrm{EC}$ for systolic and diastolic ambulatorial $\mathrm{BP}$ response, however, it is important to highlight that for diastolic BP the effects were "large" (> 0.8) for "awake", "asleep", and "24 hours" in the EC.

Despite some inconsistencies in the "effect size" outcomes for systolic and diastolic BP in the other experimental groups, the percentage of "responders" and "non-responders" and inter-individual percentage changes in the different groups (Table 4) could aid understanding. There were no "non-responders" in the EC for systolic and diastolic BP responses for "awake" (systolic and diastolic), "asleep" and "24 hours" (diastolic). These responses are important, considering that the BP response following a single bout of exercise has been considered as a simple predictive clinical tool that can help to identify and manage "high responder" and "low responder" subjects to exercise training. ${ }^{33}$

Concerning the physiological mechanisms that might be involved in this response, we hypothesized that the vascular resistance reduction due to increased $\mathrm{NO}$ concentration could potentiate the PEH. Previous studies reported that oral citrulline supplementation raises plasma arginine concentration and augments NO production by the citrulline-NO cycle. ${ }^{34,35}$ It is exactly because of this vasodilation resulting from greater NO release that citrulline oral supplementation might potentiate the $\mathrm{PEH}$ response, especially in hypertensive individuals. It is important to highlight that hypertensive individuals present 


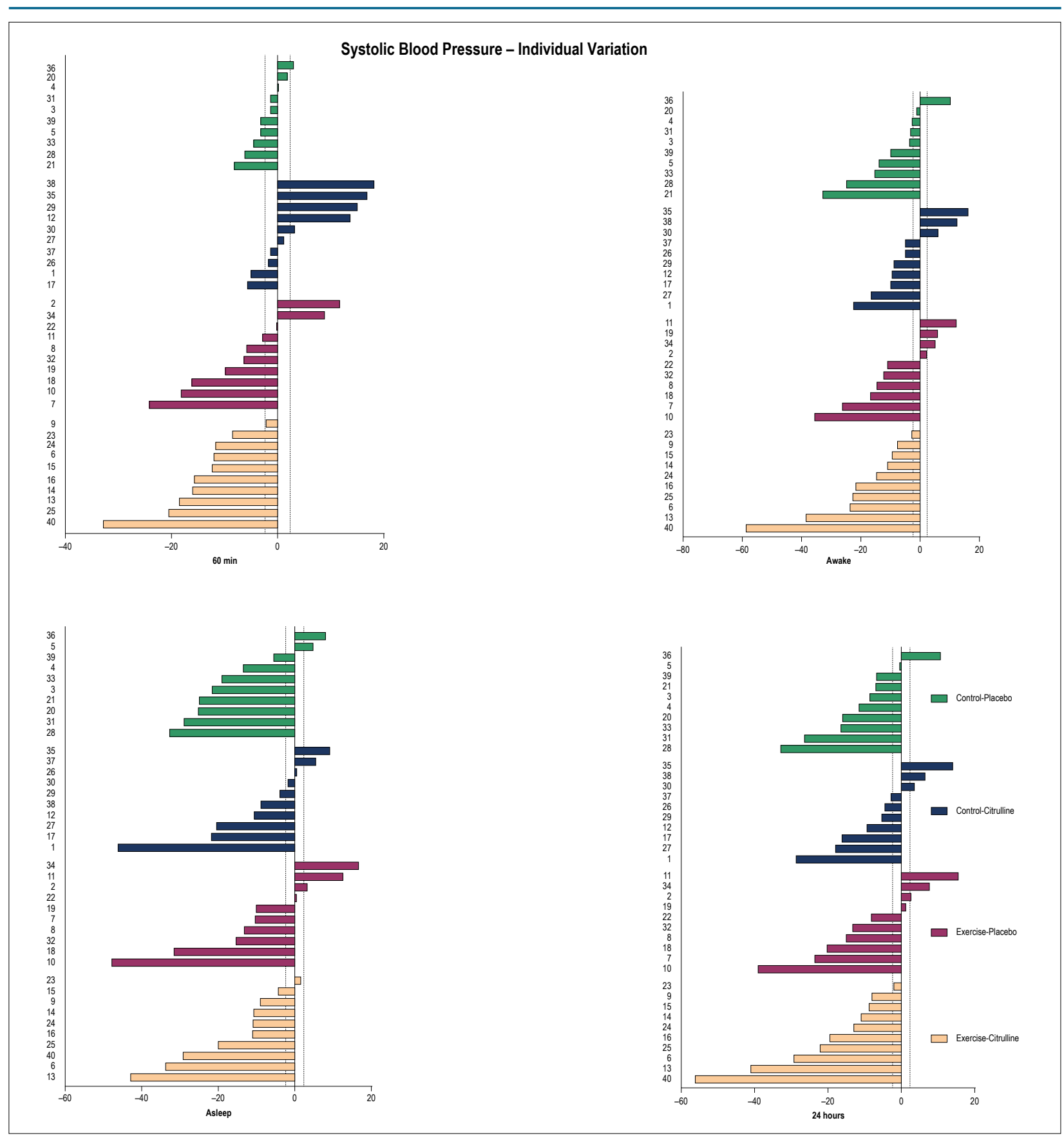

Figure 2 - Individual changes - Systolic blood pressure. Dashed line: MDC (minimal detectable change).

deficiency in peripheral vasodilation modulators, such as a compensation mechanism so autonomous nervous system can work to reduce cardiac output., ${ }^{214}$ For this reason, we expect that citrulline oral supplementation could contribute to improving the peripheral vasodilation mechanisms in hypertensive individuals, resulting in a greater magnitude and/or duration of $\mathrm{PEH}$. Unfortunately, in the present study we did not evaluate important NO biomarkers such as nitrite and nitrate.

Independent of the mechanisms that are involved in this response, the present study demonstrated that the exercise plus citrulline (intermediate of NO metabolism) caused a greater hypotensive effect and this effect can last up to 24 hours. This finding suggests that NO might be involved in this response ${ }^{36}$ and it might be over stimulated by exercise plus citrulline compared to one of them in isolation.

Concerning the $\mathrm{CP}$ and $\mathrm{CC}$ results, we expected that acute citrulline supplementation, independent of exercise, could contribute to improving hypotension effect, resulting in a lower prevalence of non-responders in comparison to CP. Contradicting our hypothesis, the results indicate that acute 


\section{Original Article}

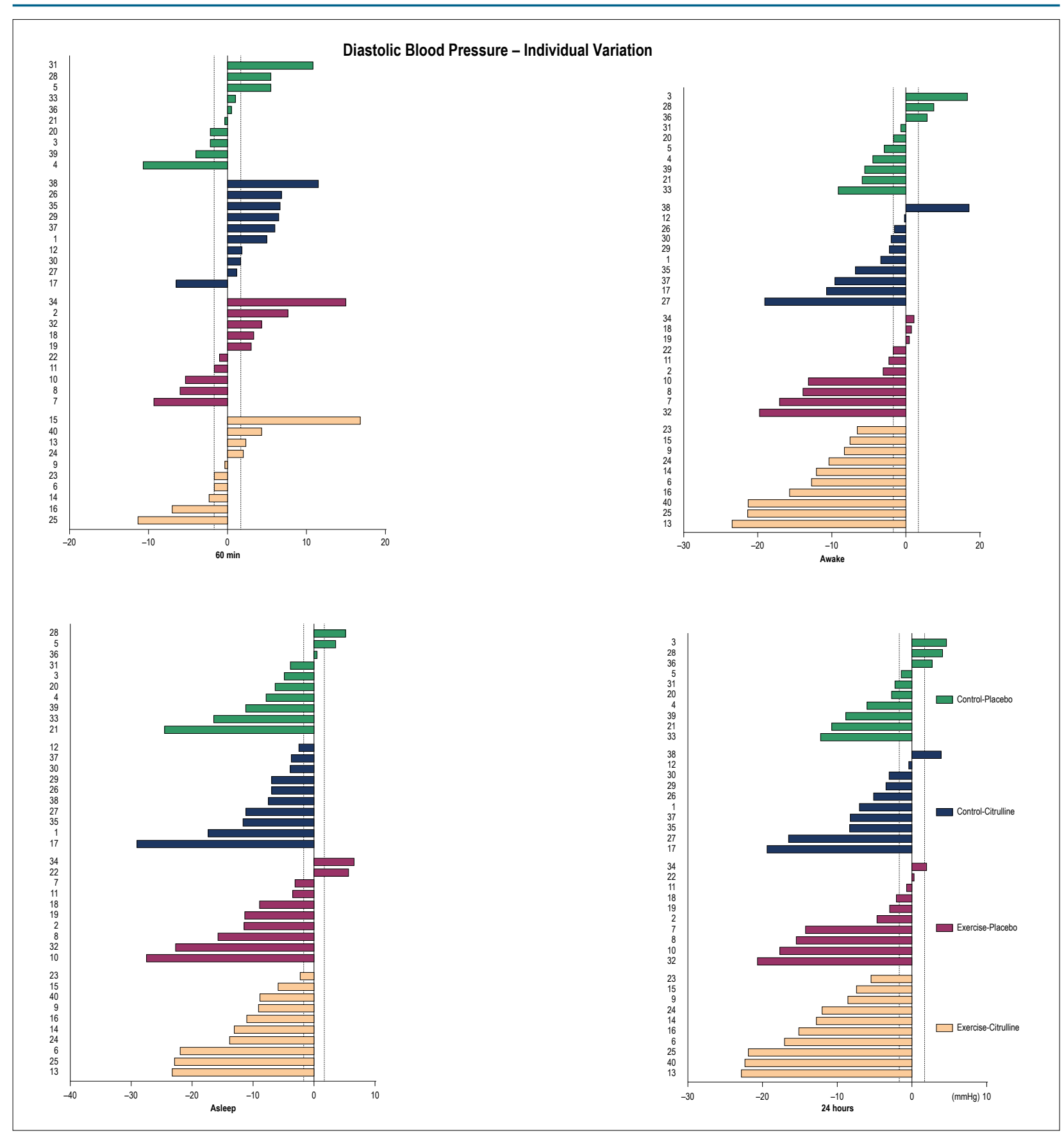

Figure 3 - Individual changes - Diastolic blood pressure. Dashed line: MDC (minimal detectable change).

citrulline supplementation was not related to lower prevalence of non-responders. Previous studies have demonstrated that the acute ingestion of citrulline increased NO synthesis, but endothelium-mediated vasodilation was not improved in older adults with heart failure. ${ }^{37}$ Recent studies have evaluated endothelial function after some days of citrulline supplementation. Bailey et al., ${ }^{38}$ found increases in nitrite levels (21\%) after 7 days of citrulline supplementation in healthy young men. Likewise, oral citrulline supplementation
(6 g/day) for 7 days increased plasma nitrate levels by $37 \%$ in middle-aged men with increased arterial stiffness. ${ }^{39}$ Evidence suggests that resting BP level may influence the hypotensive effect of citrulline supplementation. For instance, previous study found decreases in $\mathrm{BP}(7 / 3 \mathrm{mmHg})$ after 8 weeks of citrulline supplementation ( $6 \mathrm{~g} /$ day) in prehypertensive and hypertensive obese postmenopausal women. ${ }^{40}$ Therefore, we suppose that citrulline supplementation (without exercise) can induce vasodilation only after some weeks. 
Despite the interesting findings of this study, it is important to consider that there is no consensus to distinguish "responders" from "non-responders" due to the lack of agreement on whether to define "response" as a clinically relevant change or a clearly measurable change. ${ }^{41}$ Additionally, BP measurements incorporate some degree of error (instrumentation noise + biological noise), and changes can often occur due to factors independent of the intervention (biological variability). ${ }^{42}$ For this reason, the authors decided not to apply a theoretical construct (based on SD, confidence intervals, or smallest worthwhile change) to determine the extent to which changes were the direct result of the intervention or effectively "random" external causes. On the other hand, it is important to highlight that independent of the theoretical measurement error degree adopted, the EC group presented more "responsive" individuals in all hypothetical situations.

Some limitations of this study should be considered. The results from the few available studies differ considerably, making comparisons somewhat difficult. Although drug therapies were not changed during the study, patients were using different types of medication, and this factor might have affected the results. Moreover, it is recommended that future studies include NO availability measurements, such as nitrite and nitrate. These measurements associated with the evaluation of important mechanisms such as peripheral vascular resistance and cardiac output might help us to understand the citrulline action combined with exercise in hypertensives.

\section{Conclusion}

These results suggest that acute $\mathrm{CM}$ supplementation can increase the post-exercise hypotensive effects in hypertensives. In addition, the prevalence of non-responders is lower when associating aerobic exercise and CM supplementation.

\section{References}

1. Kenney MJ, Seals DR. Postexercise hypotension. Key features, mechanisms, and clinical significance. Hypertension. 1993;22(5):653-64.

2. Rondon MUB, Alves MJ, Braga AM, Teixeira OT, Barretto AC, Krieger EM, et al. Postexercise blood pressure reduction in elderly hypertensive patients. J Am Coll Cardiol. 2002;39(4):676-82.

3. Halliwill JR, Buck TM, Lacewell AN, Romero SA. Postexercise hypotension and sustained postexercise vasodilatation: what happens after we exercise? Exp Physiol. 2013;98(1):7-18.

4. Hecksteden A, Grutters T, Meyer T. Association between postexercise hypotension and long-term training-induced blood pressure reduction: a pilot study. Clin J Sport Med. 2013;23(1):58-63.

5. Kiviniemi AM, Hautala AJ, Karjalainen JJ, Piira OP, Lepojarvi S, Ukkola $\mathrm{O}$, et al. Acute post-exercise change in blood pressure and exercise training response in patients with coronary artery disease. Front Physiol. 2015 Jan $12 ; 5: 526$

\section{Clinical messages}

- There was considerable inter-individual responsiveness variability in systolic and diastolic BP responses following all experimental protocols.

- Despite the inter-individual responsiveness variability, there was a PEH potentiation by the CM supplementation.

\section{Author contributions}

Conception and design of the research, Statistical analysis and Obtaining financing: Casonatto J; Acquisition of data: Enokida DM, Grandolfi K; Analysis and interpretation of the data: Casonatto J, Enokida DM, Grandolfi K; Writing of the manuscript: Casonatto J, Enokida DM; Critical revision of the manuscript for intellectual content: Grandolfi K.

\section{Potential Conflict of Interest}

No potential conflict of interest relevant to this article was reported.

\section{Sources of Funding \\ This study was funded by FUNADESP.}

\section{Study Association}

This article is part of the thesis of master submitted by Daniel Massaharu Enokida, from Universidade Norte do Paraná.

\section{Ethics approval and consent to participate}

This study was approved by the Ethics Committee of the Universidade Norte do Paraná under the protocol CAAE: 78697617.4.0000.0108 - Appraisal number: 2.593.090. All the procedures in this study were in accordance with the 1975 Helsinki Declaration, updated in 2013. Informed consent was obtained from all participants included in the study.

6. Moreira SR, Cucato GG, Terra DF, Ritti-Dias RM. Acute blood pressure changes are related to chronic effects of resistance exercise in medicated hypertensives elderly women. Clin Physiol Funct Imaging. $2016 ; 36(3): 242-8$

7. Liu S, Goodman J, Nolan R, Lacombe S, Thomas SG. Blood pressure responses to acute and chronic exercise are related in prehypertension. Med Sci Sports Exerc. 2012;44(9):1644-52.

8. Tibana RA, de Sousa NM, da Cunha Nascimento D, Pereira GB, Thomas SG, Balsamo S, et al. Correlation between acute and chronic 24-hour blood pressure response to resistance training in adult women. Int J Sports Med. 2015;36(1):82-9

9. Costa EC, Dantas TC, de Farias Junior LF, Frazao DT, Prestes J, Moreira SR, et al. Inter- and intra-individual analysis of post-exercise hypotension following a single bout of high-intensity interval exercise and continuous exercise: a pilot study. Int J Sports Med. 2016;37(13):1038-43. 
10. Bonsu B, Terblanche E. The training and detraining effect of high-intensity interval training on post-exercise hypotension in young overweight/obese women. Eur J Appl Physiol. 2016;116(1):77-84.

11. Lima AH, Miranda AS, Correia MA, Soares AH, Cucato GG, Sobral Filho DC, et al. Individual blood pressure responses to walking and resistance exercise in peripheral artery disease patients: are the mean values describing what is happening? J Vasc Nurs. 2015;33(4):150-6.

12. Buford TW, Roberts MD, Church TS. Toward exercise as personalized medicine. Sports Med. 2013;43(3):157-65.

13. Breuillard C, Cynober L, Moinard C. Citrulline and nitrogen homeostasis: an overview. Amino Acids. 2015;47(4):685-91.

14. Floras JS, Wesche J. Haemodynamic contributions to post-exercise hypotension in young adults with hypertension and rapid resting heart rates. J Hum Hypertens. 1992;6(4):265-9.

15. Coats AJ, Conway J, Isea JE, Pannarale G, Sleight P, Somers VK. Systemic and forearm vascular resistance changes after upright bicycle exercise in man. J Physiol. 1989 Jun;413:289-98.

16. Isea JE, Piepoli M, Adamopoulos S, Pannarale G, Sleight P, Coats AJ. Time course of haemodynamic changes after maximal exercise. Eur J Clin Invest. 1994;24(12):824-9.

17. Coelho-Junior HJ, Irigoyen MC, Aguiar SDS, Goncalves IO, Camara NOS, Cenedeze MA, et al. Acute effects of power and resistance exercises on hemodynamic measurements of older women. Clin Interv Aging. 2017 Jul $11 ; 12: 1103-14$.

18. Santana HA, Moreira SR, Asano RY, Sales MM, Cordova C, Campbell CS, et al. Exercise intensity modulates nitric oxide and blood pressure responses in hypertensive older women. Aging Clin Exp Res. 2013;25(1):43-8.

19. Asano RY, Browne RAV, Sotero RC, Sales MM, Moraes JFVN, Campbell CS, et al. Cycling above rather than below lactate threshold is more effective for nitric oxide release and post-exercise blood pressure reduction in individuals with type-2 diabetes. Motriz. 2013;19(3):633-40.

20. Simoes HG, Asano RY, Sales MM, Browne RA, Arsa G, Motta-Santos D, et al. Type 2 diabetes elicits lower nitric oxide, bradykinin concentration and kallikrein activity together with higher DesArg(9)-BKand reduced post-exercise hypotension compared to non-diabetic condition. PloS One. 2013;8(11):e80348.

21. Allerton TD, Proctor DN, Stephens JM, Dugas TR, Spielmann G, Irving BA. I-Citrulline supplementation: impact on cardiometabolic health. Nutrients. 2018;10(7):pii: E921.

22. Borg G. Borg's perceived exertion and pain scales. Champaign: Human Kinetics; 1998.

23. Gordon CC, Chumlea WC, Roche AF. Stature, recumbent length, and weight. In: Lohman TG, Roche AF, Martorell R, editors. Champaign: Human Kinetics Books; 1988. 3-8 p.

24. Coleman A, Freeman P, Steel S, Shennan A. Validation of the Omron MX3 Plus oscillometric blood pressure monitoring device according to the European Society of Hypertension international protocol. Blood Press Monit. 2005;10(3):165-8

25. Pickering TG, Hall JE, Appel LJ, Falkner BE, Graves J, Hill MN, et al. Recommendations for blood pressure measurement in humans and experimental animals: part 1: blood pressure measurement in humans: a statement for professionals from the Subcommittee of Professional and Public Education of the American Heart Association Council on High Blood Pressure Research. Circulation. 2005;111(5):697-716.
26. Casonatto J, Tinucci T, Dourado AC, Polito M. Cardiovascular and autonomic responses after exercise sessions with different intensities and durations. Clinics (Sao Paulo). 2011;66(3):453-8.

27. Haley SM, Fragala-Pinkham MA. Interpreting change scores of tests and measures used in physical therapy. Phys Ther. 2006;86(5):735-43.

28. Darter BJ, Rodriguez KM, Wilken JM. Test-retest reliability and minimum detectable change using the K4b2: oxygen consumption, gait efficiency, and heart rate for healthy adults during submaximal walking. Res Q Exerc Sport. 2013;84(2):223-31.

29. Mann TN, Lamberts RP, Lambert MI. High responders and low responders: factors associated with individual variation in response to standardized training. Sports Med. 2014;44(8):1113-24.

30. Vickers AJ. The use of percentage change from baseline as an outcome in a controlled trial is statistically inefficient: a simulation study. BMC Med Res Methodol. 2001;1:6.

31. Sullivan GM, Feinn R. Using effect size-or why the $P$ value is not enough. J Grad Med Educ. 2012;4(3):279-82.

32. Lakens D. Calculating and reporting effect sizes to facilitate cumulative science: a practical primer for t-tests and ANOVAs. Front Psychol. 2013 Nov 26;4:863.

33. Luttrell MJ, Halliwill JR. Recovery from exercise: vulnerable state, window of opportunity, or crystal ball? Front Physiol. 2015 Jul 22;6:204.

34. Schwedhelm E, Maas R, Freese R, Jung D, Lukacs Z, Jambrecina A, et al. Pharmacokinetic and pharmacodynamic properties of oral L-citrulline and L-arginine: impact on nitric oxide metabolism. Br J Clin Pharmacol. 2008;65(1):51-9.

35. Mori M. Regulation of nitric oxide synthesis and apoptosis by arginase and arginine recycling. J Nutr. 2007;137(6 Suppl 2):1616S-20S.

36. McKinley-Barnard S, Andre T, Morita M, Willoughby DS. Combined L-citrulline and glutathione supplementation increases the concentration of markers indicative of nitric oxide synthesis. J Int Soc Sports Nutr. 2015 Jun 10;12:27.

37. Kim IY, Schutzler SE, Schrader A, Spencer HJ, Azhar G, Deutz NE, et al. Acute ingestion of citrulline stimulates nitric oxide synthesis but does not increase blood flow in healthy young and older adults with heart failure. Am J Physiol Endocrinol Metab. 2015;309(11):E915-24.

38. Bailey SJ, Blackwell JR, Lord T, Vanhatalo A, Winyard PG, Jones AM. I-Citrulline supplementation improves $\mathrm{O} 2$ uptake kinetics and high-intensity exercise performance in humans. J Appl Physiol (1985). 2015;119(4):385-95.

39. Ochiai M, Hayashi T, Morita M, Ina K, Maeda M, Watanabe F, et al. Shortterm effects of L-citrulline supplementation on arterial stiffness in middleaged men. Int J Cardiol. 2012;155(2):257-61.

40. Wong A, Alvarez-Alvarado S, Jaime SJ, Kinsey AW, Spicer MT, Madzima TA, et al. Combined whole-body vibration training and I-citrulline supplementation improves pressure wave reflection in obese postmenopausal women. Appl Physiol Nutr Metab. 2016;41(3):292-7.

41. Hecksteden A, Kraushaar J, Scharhag-Rosenberger F, Theisen D, Senn S, Meyer T. Individual response to exercise training - a statistical perspective. J Appl Physiol (1985). 2015;118(12):1450-9.

42. Swinton PA, Hemingway BS, Saunders B, Gualano B, Dolan E. A statistical framework to interpret individual response to intervention: paving the way for personalized nutrition and exercise prescription. Front Nutr. 2018 May 28;5:41. 
